\title{
Renormalization Group Analysis of Nonlinear Diffusion Equations with Periodic Coefficients
}

\author{
Gastão A. Braga* Frederico Furtado ${ }^{\dagger}$ \\ Jussara M. Moreira* Leonardo T. Rolla*
}

October, 2003

\begin{abstract}
In this paper we present an efficient numerical approach based on the Renormalization Group method for the computation of self-similar dynamics. The latter arise, for instance, as the long-time asymptotic behavior of solutions to nonlinear parabolic partial differential equations. We illustrate the approach with the verification of a conjecture about the long-time behavior of solutions to a certain class of nonlinear diffusion equations with periodic coefficients. This conjecture is based on a mixed argument involving ideas from homogenization theory and the Renormalization Group method. Our numerical approach provides a detailed picture of the asymptotics, including the determination of the effective or renormalized diffusion coefficient.
\end{abstract}

This preprint has the same numbering of sections, equations and figures as the published article "Multiscale Model. Simul. 1 (2003), p. 630-644."

Key words. renormalization group, partial differential equations, multiple scale problems, asymptotic behavior.

AMS subject classifications. 35K55, 35B40, 35B33, 35B27, 74S20

DOI. $10.1137 /$ S1540345902416600

This work was supported by CNPq-Brazil and NSF under Grant INT-0104529.

*Departamento de Matemática - UFMG - Brazil.

${ }^{\dagger}$ Department of Mathematics - University of Wyoming - USA. 


\section{Introduction}

The time evolution of many non-equilibrium physical systems is well described by nonlinear partial differential equations (PDEs). It is frequently observed that under suitable, but fairly general, conditions the time evolution of such systems becomes asymptotically self-similar. Within the PDE framework, this assertion amounts to the observation that the PDEs in question have solutions that behave asymptotically as

$$
u(x, t) \sim \frac{1}{t^{\alpha}} \phi\left(\frac{x}{t^{\beta}}\right), \quad \text { as } \quad t \rightarrow \infty .
$$

It turns out that often such a self-similar behavior possesses a great deal of universality: the scaling exponents $\alpha$ and $\beta$ and most aspects of the profile function $\phi(\cdot)$ are independent of initial conditions (within a suitable class) or, more strikingly, even of the form of the equations.

Universal behavior is a central issue in the study of critical phenomena in equilibrium statistical mechanics and quantum field theory. Using the Renormalization Group (RG) approach [11, 18, 14], physicists predict critical exponents and determine the universality class of a variety of models. In the early 90's, Goldenfeld, Oono and collaborators (see [12] and references therein) developed a perturbative renormalization group method for PDEs and used it to compute perturbatively the similarity exponents in the difficult and most frequently encountered case of self-similarity of the second kind (in Barenblatt's classification [1]). Later, Bricmont, Kupiainen and Lin [6, 1] introduced a non-perturbative renormalization group approach. A numerical renormalization group algorithm was developed at the same time by Chen and Goldenfeld [8].

In this paper we combine the numerical approach of Chen and Goldenfeld with the constructive approach of Bricmont et al. to develop a computationally efficient RG algorithm for the calculation of self-similar dynamics. This algorithm is then used to produce a detailed picture of the asymptotic dynamics of certain nonlinear diffusion equations with periodic coefficients; in particular, this algorithm allows the investigation of which aspects of these dynamics are universal and which are not. A similar point of view was adopted by some of the authors [4] to study a modification of Barenblatt's equation. Isaia [13] also used the same approach to study other classes of problems including some with logarithmic corrections to the time decay.

Our purpose in writing this paper is threefold. Firstly, we conjecture the long-time asymptotic behavior of a class of PDEs. Secondly, following the strategy of [4, 13], we present a systematic numerical approach, based on the RG ideas, to study self-similar dynamics. Finally, we point out a connection between the renormalization group and homogenization theory [2]. Homogenization theory has been applied with success to critical lattice field theories [16], where a RG approach should work. Here, in addition to adopting a reverse point of view and showing that the $R G$ approach succeeds in 
problems typically analyzed with homogenization theory, we further point out that the RG approach gives more general results; see below.

Numerical procedures based on rescaling, and thus similar in spirit to the RG approach presented here, have previously been developed [15, 3] and used to study solutions which blow up in finite time (as, for example, in the case of certain reaction-diffusion equations and the cubic nonlinear Schrödinger equation). Such procedures exploit the known self-similar structure of the solutions under study to define the appropriate rescalings. Recently, versatile and more efficient versions of such procedures were developed [17, 10] and employed for the computation of solutions which blow up at multiple points. Yet, the $R G$ procedure presented here is unique in exploiting fixed points. The central feature of this procedure is that the rescalings are determined dynamically, not by any a priori knowledge of the scale invariance of the solutions. As a result, the procedure can be used to investigate self-similar asymptotics of both the first and second (anomalous) kind. On the other hand the current implementation of the procedure that we present here is not appropriate for studying blow-up problems.

This paper proceeds as follows: in Section 2 we define the class of initial value problems we study and state the conjecture we make about the long time behavior of their solutions. In Section 3 we present a non-rigorous argument to motivate this conjecture. In Section 4 we explain how we have implemented our modification of the numerical renormalization group scheme of Chen and Goldenfeld [8] to the oscillating equation (2.1). In Section 5 we present numerical results that corroborate our conjecture. We also present studies to validate our numerical procedure, including the computation of the critical exponent $\alpha$ for solutions of Barenblatt's equation to illustrate the versatility of our procedure in computing anomalous behavior. Finally, in Section 6 we present numerical evidence supporting a similar conjecture for "relevant" perturbations (see the Remark in the next section), make some comments and discuss further developments.

\section{Asymptotics of oscillating equations}

Consider the following initial value problem (IVP):

$$
\begin{gathered}
u_{t}=[1+\mu g(x)] u_{x x}+\lambda F\left(u, u_{x}, u_{x x}\right) \quad x \in \mathbb{R}, t>1, \\
u(x, 1)=f(x), \quad x \in \mathbb{R} .
\end{gathered}
$$

Here $\mu$ and $\lambda$ are real parameters; $\mu$ is such that $(1+\mu g(x))>0$ for all $x \in \mathbb{R} ; g(x)$ is a smooth periodic function with zero mean; $f(x)$ is a smooth and rapidly decaying initial condition; $F(u, v, w)$ is an analytic function of $u, v$ and $w$ around $u=v=w=0$ :

$$
F(u, v, w)=u^{a} v^{b} w^{c}+\sum_{\{i>a, j>b, k>c\}} c_{i j k} u^{i} v^{j} w^{k} .
$$


Let $T$ be the period of the function $g$ and let $H(g, \mu)$ denote the harmonic mean of $1+\mu g(x)$ :

$$
H(g, \mu) \equiv\left[\frac{1}{T} \int_{0}^{T} \frac{1}{1+\mu g(x)} d x\right]^{-1} .
$$

We conjecture the following.

Conjecture 2.1 Let $u(x, t)$ be the solution of the IVP (2.1). If the lowest order exponents of the power series expansion of $F$ satisfy the inequality $a+2 b+3 c-3>0$ and if the initial condition $f$ is small enough, then there is a constant $A$, which usually depends on $f, g, \mu, \lambda$ and $F$, such that

$$
t^{\alpha} u(\sqrt{t} x, t) \rightarrow \frac{A}{\sqrt{4 \pi \sigma}} e^{-\frac{x^{2}}{4 \sigma}} \quad \text { as } \quad t \rightarrow \infty,
$$

with $\alpha=1 / 2$ and $\sigma=H(g, \mu)$.

As we shall see, this conjecture arises from formal renormalization group arguments applied to (2.1). These arguments are based on the methods developed by Goldenfeld, Oono and collaborators [12] and by Bricmont, Kupiainen and Lin [6, 7]. Implementing a modification of the RG-based numerical algorithm of Chen and Goldenfeld [8], we verify the validity of the conjecture.

Remark: Let $d_{F} \equiv a+2 b+3 c-3$. Bricmont et al. [7] classify the perturbations $F$ (see Eq. (2.1) ) as irrelevant if $d_{F}>0$, marginal if $d_{F}=0\left(F=u u_{x}, u_{x x}\right.$ or $\left.u^{3}\right)$ and relevant if $d_{F}<0\left(F=u_{x}\right.$ or $\left.u^{a}\right)$. Conjecture 2.1 is stated for irrelevant perturbations but similar conjectures hold for the marginal and relevant cases, although care is needed in order to avoid blow up at finite time. In particular, if $\lambda F=-u^{a}+$ irrelevant perturbations, $1<a<3$, then, instead of (2.3) we expect that

$$
t^{\frac{1}{a-1}} u(\sqrt{t} x, t) \rightarrow f_{a}\left(\frac{x}{\sqrt{\sigma}}\right) \quad \text { as } \quad t \rightarrow \infty
$$

where $f_{a}$ is a function such that

$$
u(x, t) \equiv \frac{1}{t^{\frac{1}{a-1}}} f_{a}\left(\frac{x}{t^{1 / 2}}\right)
$$

is a scale invariant solution to Eq. (2.1) with $\mu=0$ and $\lambda F=-u^{a}$, see [5, 6, 7]. In Section 6 we present numerical results supporting this conjecture. Due to logarithmic corrections, our numerical approach can not handle marginal perturbations efficiently. We point out that logarithmic corrections have been obtained using another version of the numerical RG in [13].

We emphasize the following aspects of (2.3): 
1. the exponent $\alpha$ is universal: $\alpha=1 / 2$ independently of the initial condition $f$, the periodic function $g$, the parameters $\mu$ and $\lambda$ and the perturbation $F\left(u, u_{x}, u_{x x}\right)$ provided that $a+2 b+3 c-3>0$;

2. the effective diffusion coefficient $\sigma$ depends on $\mu$ and $g: \sigma=H(g, \mu)$;

3. in general, the prefactor $A$ depends on the initial condition $f$, on the perturbations $F\left(u, u_{x}, u_{x x}\right)$ and $g(x)$ and on the parameters $\mu$ and $\lambda$.

Conjecture 2.1 can be understood as a statement about the universality class of Gaussian fixed points of a renormalization group transformation. It furnishes a classification of the nonlinear terms that can be added to the linear oscillating equation without changing the long-time asymptotics of the associated solutions. The work of Duro and Zuazua [9] can also be interpreted within this framework. They have considered the equation

$$
u_{t}-\operatorname{div}(a(\vec{x}) \nabla u)=\vec{d} \cdot \nabla\left(|u|^{q-1} u\right) \text { in } \mathbb{R}^{N} \times(0, \infty),
$$

where $\vec{d} \in \mathbb{R}^{N}$ and $a(\vec{x})$ is a smooth, symmetric and periodic matrix. Using homogenization theory, they have proved, among other results, the asymptotic result (2.3) whenever $q>1+1 / N$. Although we do not prove it, our conjecture indicates that Duro and Zuazua's results should hold for other non-linear equations.

\section{$3 \quad$ Renormalization group analysis}

In this section we present the formal RG arguments which led to the aforementioned conjecture. In the sequel, we assume that the perturbation $F\left(u, u_{x}, u_{x x}\right)$ in Eq. (2.1) is of the form $u^{a} u_{x}^{b} u_{x x}^{c}$. Later, it will become clear that this assumption constitutes no loss of generality since the higher powers of $F$ are "irrelevant" in the RG sense. The renormalization group approach that we employ in this paper is simply the integration of the equation followed by a rescaling. To explain this idea, we need some preliminary notions.

Let $u$ be a real-valued function of $(x, t) \in \mathbb{R} \times \mathbb{R}_{+}$. For a fixed $L>1$ and sequences of positive scaling exponents, $\left\{\alpha_{n}\right\}_{n=1}^{\infty}$ and $\left\{\beta_{n}\right\}_{n=1}^{\infty}$, define a sequence $\left\{u_{n}\right\}_{n=0}^{\infty}$ of rescaled functions inductively by $u_{0}=u$ and, for $n \geq 1$,

$$
u_{n}(x, t)=L^{\alpha_{n}} u_{n-1}\left(L^{\beta_{n}} x, L t\right)=L^{\alpha_{n}+\cdots+\alpha_{1}} u\left(L^{\beta_{n}+\cdots+\beta_{1}} x, L^{n} t\right) .
$$

If the original function $u$ is a global solution to Eq. (2.1), then a direct calculation reveals that $u_{n}$ satisfies the renormalized initial value problem:

$$
\partial_{t} u_{n}=\chi_{n}\left[1+\mu g\left(\omega_{n} x\right)\right] \partial_{x}^{2} u_{n}+\lambda_{n} u_{n}^{a}\left(\partial_{x} u_{n}\right)^{b}\left(\partial_{x}^{2} u_{n}\right)^{c},
$$




$$
u_{n}(x, 1)=f_{n}(x)
$$

Here

$$
\chi_{n}=L^{n-2\left(\beta_{n}+\cdots+\beta_{1}\right)}, \quad \omega_{n}=L^{\beta_{n}+\cdots+\beta_{1}},
$$

and

$$
\lambda_{n}=\lambda L^{n-(b+2 c)\left(\beta_{n}+\cdots+\beta_{1}\right)+(1-a-b-c)\left(\alpha_{n}+\cdots+\alpha_{1}\right)},
$$

and the initial data $f_{n}$ is

$$
f_{n}(x)=L^{\alpha_{n}+\cdots+\alpha_{1}} u\left(L^{\beta_{n}+\cdots+\beta_{1}} x, L^{n}\right) .
$$

The renormalization group transformation of Bricmont et al., which we denote as $R_{L}$, is now introduced. This transformation acts on the space of initial conditions and is defined having the linear diffusion equation

$$
u_{t}=u_{x x}
$$

in mind. As explained in [6, 7], the study of the long time asymptotics of solutions to (2.1) with $\mu=0$ is equivalent to studying the fixed points, and their basins of attraction (i.e. universality classes), of the transformation $R_{L}$. The $\mathrm{RG}$ transformation is designed so that its fixed points are the similarity solutions to (3.5). Below we adopt the same strategy and use $R_{L}$ to study the long time asymptotics of solutions to the oscillating equation (2.1).

To define $R_{L}$, take $L>1$ and set $\alpha_{i}=\beta_{i}=1 / 2$ for all $i \geq 1$. With this choice, $\chi_{n}=1$, $\omega_{n}=L^{n / 2}, \lambda_{n}=\lambda L^{n[3-(a+2 b+3 c)] / 2}$ and the IVP (3.2) becomes

$$
\begin{gathered}
\partial_{t} u_{n}=\left[1+\mu g\left(L^{n / 2} x\right)\right] \partial_{x}^{2} u_{n}+\lambda L^{n[3-(a+2 b+3 c)] / 2} u_{n}^{a}\left(\partial_{x} u_{n}\right)^{b}\left(\partial_{x}^{2} u_{n}\right)^{c}, \\
u_{n}(x, 1)=f_{n}(x) .
\end{gathered}
$$

If $u_{n}(x, t)$ is the solution to the IVP (3.6) for $t \in[1, L], R_{L}$ is defined as

$$
\left(R_{L} f_{n}\right)(x)=L^{1 / 2} u_{n}\left(L^{1 / 2} x, L\right) \equiv f_{n+1}(x) .
$$

$R_{L}$ depends on $n$ but we do not write explicitly this dependence to simplify the notation. Notice that $R_{L}$ has the semigroup property:

$$
R_{L}^{n} f(x)=R_{L^{n}} f(x)=L^{n / 2} u\left(L^{n / 2} x, L^{n}\right), \quad n=1,2, \ldots
$$

This fact allows us to investigate the limit (2.3), with $t=L^{n}$, by iterating $R_{L}$, and this is how we proceed. We start our analysis by reviewing the results of Bricmont et al. [6, 7] when $\mu=0 \neq \lambda$ : 
1. let $\mathcal{R}$ denote the linearized $R G$ transformation (take $\mu=\lambda=0$ in equation (2.1)).

One can check that any multiple of the Gaussian distribution

$$
\phi_{*}(x) \equiv \frac{e^{-\frac{x^{2}}{4}}}{\sqrt{4 \pi}}
$$

is a fixed point of $\mathcal{R} . \phi_{*}(x)$ is called the Gaussian fixed point;

2. any smooth function $g$ decaying sufficiently fast and with $\hat{g}(0)=0$, where $\hat{g}(k)$ denotes the Fourier transform of $g(x)$, contracts to zero under the action of $\mathcal{R}$ :

$$
\|\mathcal{R} g\|_{\infty} \leq \frac{C}{L}\|g\|_{\infty}<\|g\|_{\infty}
$$

if $L>C$;

3. any function $f$ can be written as its projection onto $\phi_{*}$ plus a remainder $g$ with $\hat{g}(0)=0: f=\hat{f}(0) \phi_{*}+g$

4. write $R_{L}^{n} f(x)$ as $R_{L}^{n} f(x)=A_{n} \phi_{*}(x)+g_{n}(x)$, where $\hat{g_{n}}(0)=0$. Then, if the exponents $a, b$ and $c$ are such that $[(a+2 b+3 c)-3]>0, A_{n} \rightarrow A$ and $g_{n}(x) \rightarrow 0$ as $n \rightarrow \infty$.

In other words, Bricmont et al. proved that the long-time behavior of the solution $u(x, t)$ to the IVP (2.1) with $\mu=0$ is given by the solution of

$$
\begin{gathered}
u_{t}=u_{x x}, \\
u(x, 1)=A \phi_{*}(x) .
\end{gathered}
$$

Now consider the case $\mu \neq 0=\lambda$. Using homogenization theory [2], one can conclude that the limit (2.3) exists. This is done as follows. After $n$ RG iterations of equation (2.1) with $\lambda=0$ we obtain:

$$
\begin{gathered}
\partial_{t} u=\left[1+\mu g\left(L^{n / 2} x\right)\right] \partial_{x}^{2} u \\
u_{n}(x, 1)=f_{n}(x) .
\end{gathered}
$$

Take $\epsilon_{n}^{-1}=L^{n / 2}$. Observing that $\epsilon_{n} \rightarrow 0$ as $n \rightarrow \infty$, we see that studying the asymptotics of the solution to IVP (3.8) as $n \rightarrow \infty$ is equivalent to studying the asymptotics as $\epsilon_{n} \rightarrow 0$, a situation which can be dealt with by homogenization theory which says that the long time behavior is governed by a multiple of the solution to

$$
\begin{gathered}
\partial_{t} u=\sigma u_{x x} \\
u(x, 1)=\phi_{\sigma}(x),
\end{gathered}
$$


where $\sigma=H(g, \mu)$ is the harmonic mean defined by equation (2.2) and

$$
\phi_{\sigma}(x)=\frac{e^{-\frac{x^{2}}{4 \sigma}}}{\sqrt{4 \pi \sigma}} .
$$

For the general case $\mu \neq 0 \neq \lambda$, the RG iteration of the IVP (2.1) is represented by the IVP (3.6). As $n \rightarrow \infty$, the second term on the right hand side of Eq. (3.6) is expected to be driven to zero if $[(a+2 b+3 c)-3]>0$. Writing $R_{L}^{n} f(x)$ as $R_{L}^{n} f(x)=A_{n} \phi_{\sigma}(x)+g_{n}(x)$, with $\hat{g_{n}}(0)=0$, we also expect that $A_{n} \rightarrow A$ and that $g_{n}(x) \rightarrow 0$ as $n \rightarrow \infty$. Therefore, for large values of $n$, we expect the solution to the IVP (2.1) to behave as the solution to the IVP (3.9), with $A=\lim A_{n}$ and $\sigma=H(g, \mu)$.

\section{The numerical procedure}

The RG-based numerical procedure for the integration of the IVP (2.1) is now introduced. The renormalization procedure constructs the sequence of renormalized IVP (3.2) (viz. the sequences $\left\{\alpha_{n}\right\},\left\{\beta_{n}\right\}$ and $\left\{f_{n}\right\}$ ) satisfied by the solution of (2.1) in the following steps.

Start with $f_{0}=f$, the initial condition of the IVP (2.1). For $n=0,1,2, \ldots$

1. Evolve the initial data $f_{n}$ forward, from the initial time $t=1$ to $t=L$, with $L>1$ arbitrary but fixed, using Eq. (3.2), to obtain the solution $u_{n}(x, L)$.

2. Compute the scaling exponents $\alpha_{n+1}, \beta_{n+1}$ as

$$
L^{\alpha_{n+1}}=\frac{u_{n}(0,1)}{u_{n}(0, L)}=\frac{f_{n}(0)}{u_{n}(0, L)}, \quad \beta_{n+1}=\frac{1}{2} .
$$

(This choice will be discussed shortly.)

3. Define $f_{n+1}(x)=L^{\alpha_{n+1}} u_{n}\left(L^{\beta_{n+1}} x, L\right)$.

Notice that if $f_{0}=f$, then (3.1) and the definition of $f_{n}$ imply that

$$
f_{n}(x)=L^{\alpha_{n}} u_{n-1}\left(L^{\beta_{n}} x, L\right)=L^{\alpha_{n}+\cdots+\alpha_{1}} u\left(L^{\beta_{n}+\cdots+\beta_{1}} x, L^{n}\right),
$$

where $u\left(\cdot, L^{n}\right)$ is the solution of the initial value problem (2.1) at time $t=L^{n}$.

A simple consideration of (4.2) uncovers the relationship between the limiting behavior of $\left\{f_{n}\right\}$ as $n \rightarrow \infty$ and the long time asymptotics of $u$. To wit, rewrite (4.2) as

$$
u\left(x, L^{n}\right)=\frac{A_{n}}{L^{n \alpha_{n}}} f_{n}\left(B_{n} \frac{x}{L^{n \beta_{n}}}\right),
$$


where

$$
A_{n}=L^{n \alpha_{n}-\left(\alpha_{n}+\cdots+\alpha_{1}\right)}, \quad B_{n}=L^{n \beta_{n}-\left(\beta_{n}+\cdots+\beta_{1}\right)} .
$$

Thus, if the limits $A_{n} \rightarrow A, B_{n} \rightarrow B, \alpha_{n} \rightarrow \alpha, \beta_{n} \rightarrow \beta$ and $f_{n} \rightarrow \phi$ as $n \rightarrow \infty$ are attained, we might expect that

$$
L^{n \alpha} u\left(L^{n \beta} x, L^{n}\right)=\frac{A_{n}}{L^{n\left(\alpha_{n}-\alpha\right)}} f_{n}\left(B_{n} \frac{x}{L^{n\left(\beta_{n}-\beta\right)}}\right) \rightarrow A \phi(B x) \quad n \rightarrow \infty .
$$

This limit, in turn, establishes the self-similarity of the long time asymptotics of $u$.

It is the numerical algorithm consisting of steps $1-3$ above supplemented by the calculation of the prefactors $\left\{A_{n}\right\}$ and $\left\{B_{n}\right\}$ that we use to verify the Conjecture 2.1 about the asymptotic behavior of solutions to (2.1). We remark that this procedure yields a very detailed picture of the asymptotics. In particular, it seems ideal to study which aspects of the dynamics are universal and which depend on parameters and initial conditions. A number of comments are in order.

The rationale behind the idea we use to compute $\left\{\alpha_{n}\right\}$ in step 2 is the reputed self-similar asymptotic dynamics we want to compute: in the self-similar regime, the solution $u$ at $x=0$, behaves as

$$
u(0, t) \sim \operatorname{const} t^{-\alpha} \text {. }
$$

Thus, given the relation between $u_{n}$ in the time interval $t \in[1, L]$ and $u$ in $t \in\left[L^{n}, L^{n+1}\right]$ (c.f. (3.1)), we expect that, as $n \rightarrow \infty, \alpha_{n}$ approaches $\alpha$. Whereas the determination of the exponents $\alpha_{n}$ can always be performed as in step 2 above, the determination of the exponents $\beta_{n}$ is problem dependent. It usually involves a scaling relation between the exponents $\alpha_{n}$ and $\beta_{n}$ so that certain (a priori chosen) parts of the differential operator remain invariant under the rescaling of step 3. To illustrate this point, consider the initial value problem (2.1) with $F\left(u, u_{x}, u_{x x}\right)=u^{a} u_{x}^{b} u_{x x}^{c}$. If $\beta_{n}$ is chosen to be $1 / 2$, then the linear operator

$$
u_{t}=u_{x x}
$$

remains invariant under the scaling defined in step 3. In this way, the dynamics associated with (4.3) can be explored. On the other hand, if $\beta_{n}$ is chosen to satisfy the scaling relation (c.f. equation (3.3) $)$

$$
1-(b+2 c) \beta_{n}+(1-a-b-c) \alpha_{n}=0,
$$

then it is the nonlinear operator

$$
u_{t}=\lambda u^{a} u_{x}^{b} u_{x x}^{c}
$$

that remains invariant. In this case, it is the dynamics of (4.5) that can be explored. In the $\mathrm{RG}$ language, the choice $\beta_{n}=1 / 2$ focuses the attention on the dynamics of (4.3) and, as such, is suitable for the investigation of its irrelevant and marginal perturbations. 
This is done in Section 5 for the Equation (2.1). When the perturbation is relevant, then a new scaling relation may be needed. For instance, with the choice (4.4), the nonlinear operator $u_{t}=u^{a} u_{x}^{b} u_{x x}^{c}$ remains invariant (under the associated scaling). Therefore this is the appropriate scaling (which then defines an appropriate RG map) if we want to study irrelevant and marginal perturbations to the nonlinear operator $u_{t}=u^{a} u_{x}{ }^{b} u_{x x}{ }^{c}$. In Section 6 we study a case when both the Laplacian and the nonlinear term become marginal under an appropriate scaling (see the remark on Section 2 and the limiting behavior given by (2.4)) .

Finally, the spatial scaling in step 3 can be realized in two different ways: by rescaling the mesh size $\Delta x$ without changing the discrete sites $j=0,1, \ldots$, so that after one iteration the new mesh size is $(\Delta x)_{1}=L^{-\beta_{1}} \Delta x$ and the new mesh points are located at $x=j L^{-\beta_{1}} \Delta x$; this is the approach adopted in Ref. [8]; or by rescaling the discrete sites while keeping the mesh size fixed, so that after one iteration the new discrete sites

$L^{-\beta} j$ are located at $x=L^{-\beta_{1}} j \Delta x$. In this approach, the values of the solution $u$ at the fixed mesh points $x=j \Delta x$ have to be interpolated from the data given at the (new) discrete sites in each iteration.

In our investigation of the asymptotics of problem (2.1) we have used the first approach. The successive spatial scalings performed in step 3 change the frequency of the periodic diffusion coefficient. It is actually this mechanism that leads to a renormalization of the diffusion coefficient in the long time regime. Thus, to resolve properly the effects of the oscillating diffusion, the mesh size has to be shrunk at the same rate as the frequency is increased.

\section{$5 \quad$ Numerical results}

For the discretization of the governing PDE, any appropriate scheme can be employed. We chose a simple explicit finite difference scheme that combines Euler's method for the time discretization with the standard three-point formula for the discretization of the Laplacian operator and centered differences for the first order spatial derivatives. Given the stability constraints, the resulting scheme is second order accurate.

To eliminate the need of numerical boundary conditions in our computations of purely initial value problems with compactly supported initial data, additional grid points with associted zero-valued data were added at the boundaries of the computational domain before each timestep was performed. In all simulations 27 grid points were used for the discretization of the initial data in $-5 \leq x \leq 5$ (initial computational domain). The time step was dynamically chosen to satisfy the stability condition $(1+\max |\mu g|) \Delta t \leq$ $C \Delta x^{2}$ (the grid spacing $\Delta x$ shrinks as the renormalization procedure is iterated). The constant $C$ was chosen to be 0.45 . 
Table 1: Description of the numerical simulations, with $L=1.021$ and $\beta=0.5$

\begin{tabular}{||r|r|r|r|r|r|r|r||}
\hline \hline$n$ & $\mu$ & $g$ & $\lambda$ & $a$ & $b$ & $c$ & $f$ \\
\hline \hline 1 & .1 & $g_{1}$ & 0 & & & & $f_{1}$ \\
\hline 2 & .1 & $g_{1}$ & 0 & & & & $f_{2}$ \\
\hline 3 & -.15 & $g_{1}$ & 0 & & & & $f_{1}$ \\
\hline 4 & .1 & $g_{1}$ & 0 & & & & $f_{3}$ \\
\hline 5 & .1 & $g_{1}$ & .1 & 4 & 0 & 0 & $f_{1}$ \\
\hline 6 & .1 & $g_{1}$ & .1 & 2 & 1 & 0 & $f_{1}$ \\
\hline 7 & .1 & $g_{2}$ & .3 & 8 & 0 & 0 & $f_{3}$ \\
\hline 8 & .8 & $g_{1}$ & 0 & & & & $f_{1}$ \\
\hline 9 & .1 & $g_{3}$ & 0 & & & & $f_{1}$ \\
\hline 10 & .8 & $g_{3}$ & 0 & & & & $f_{1}$ \\
\hline 11 & 0 & & 0 & & & & $f_{1}$ \\
\hline 12 & 0 & & 0 & & & & $f_{2}$ \\
\hline 13 & 0 & & 0 & & & & $f_{3}$ \\
\hline 14 & .1 & $g_{1}$ & .1 & 1 & 1 & 1 & $f_{1}$ \\
\hline 15 & .6 & $g_{3}$ & .1 & 0 & 1 & 1 & $f_{2}$ \\
\hline \hline
\end{tabular}

Table 1 summarizes our numerical simulations: the first column is the simulation number; the other columns specify the parameters $\mu$ and $\lambda$, the exponents $a, b$ and $c$ and the functions $f$ and $g$ appearing in the IVP (3.2) (functions $f_{i}$ and $g_{i}, i=1,2,3$ are specified in Figures 1 and 2). In all simulations, the scaling factor $L$ was chosen to be $L=1.021$. Although, in principle, any value of $L>1$ can be used, for a too large value of $L$, a large number of time steps per $R G$ iteration would be required, and the system would shrink too quickly (due to the spatial rescaling); for a very small $L$, a large number of RG iterations would be necessary to produce good enough accuracy.

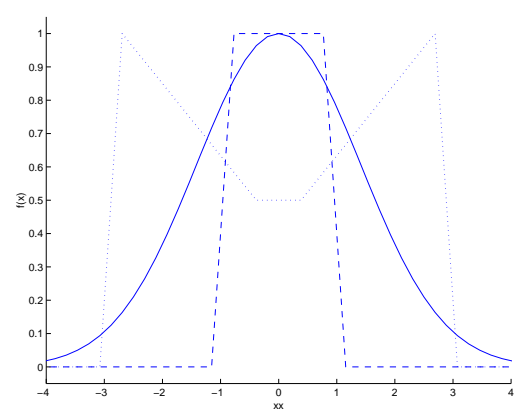

Figure 1: Initial conditions $f_{1}$ (continuous line), $f_{2}$ (dashed line), $f_{3}$ (dotted line).

We now discuss some of the simulations. In order to validate the numerical procedure and to illustrate its accuracy, efficiency and the amount of detailed information it provides, the algorithm was applied to the heat equation $(\mu=0=\lambda)$, an equation whose 


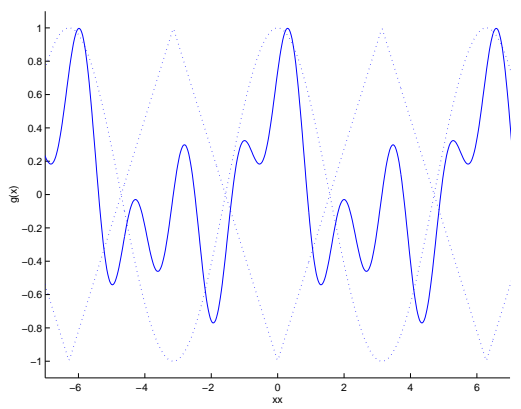

Figure 2: Periodic functions: $g_{1}(x)=\cos (x), g_{2}(x)=[\cos (x)+\sin (2 x)+\cos (4 x)] / 2.72$, $g_{3}(x)=1-2|x / \pi \bmod 2-1|$

asymptotic behavior is well known (simulations number 11, 12, 13). Figures 3, 4, and 5 refer to this study, as explained below. Also, simulations for Barenblatt's equation

$$
u_{t}=\left(1+\epsilon H\left(-u_{t}\right)\right) u_{x x}
$$

where $H(u)$ is the Heaviside function, were performed for different values of $\epsilon$. Figure 6 shows the anomalous exponent $\alpha$ computed by our simulations, compared with the first-order approximation [12]

$$
\alpha=\frac{1}{2}+\frac{\epsilon}{\sqrt{2 \pi e}}+O\left(\epsilon^{2}\right) .
$$

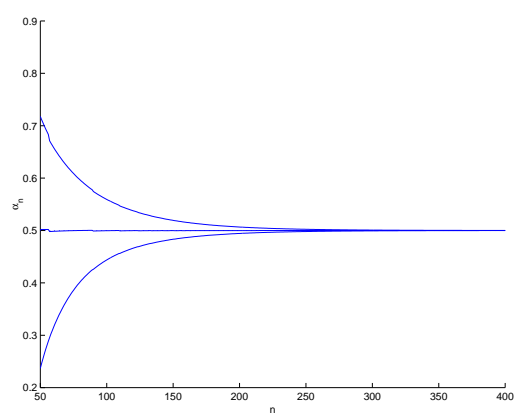

Figure 3: Validation simulation (heat equation): plot of $\alpha_{n} \times n$, for simulations 11, 12 and 13 , verifying that $\alpha^{*}=\lim \alpha_{n}$ is universal with respect to initial conditions.

Figure 3 depicts the convergence of the computed exponent $\alpha_{n}$ to the theoretical value $\alpha=1 / 2$ as the numerical RG map is iterated for several distinct initial conditions. This illustrates the universality of $\alpha$ with respect to sufficiently localized (e.g. with 


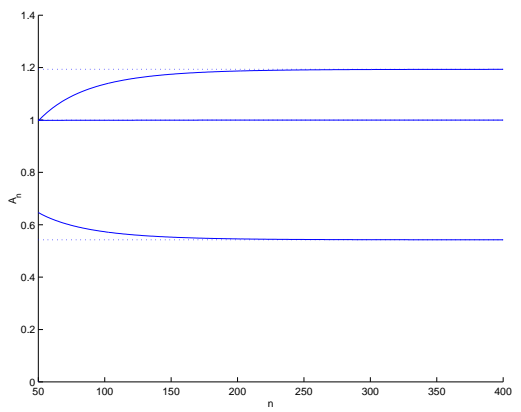

Figure 4: Validation simulation (heat equation): plot of $A_{n} \times n$, for simulations 11,12 and 13 , verifying that $A_{n} \rightarrow A$ and that $A$ is mass dependent.

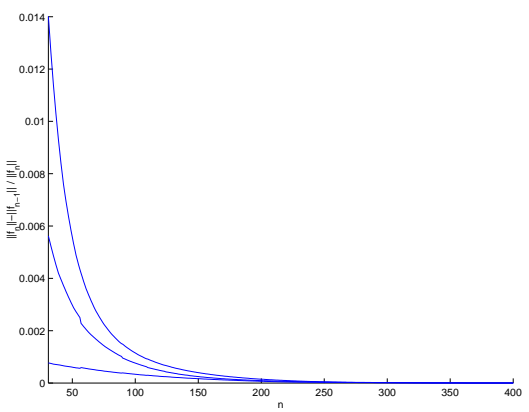

Figure 5: Validation simulation (heat equation): plot of $\left\|f_{n}-f_{n-1}\right\| /\left\|f_{n}\right\| \times n$, for simulations 11,12 and 13 , verifying that the profile function $f_{n}$ converges upon the iteration of the numerical RG map.

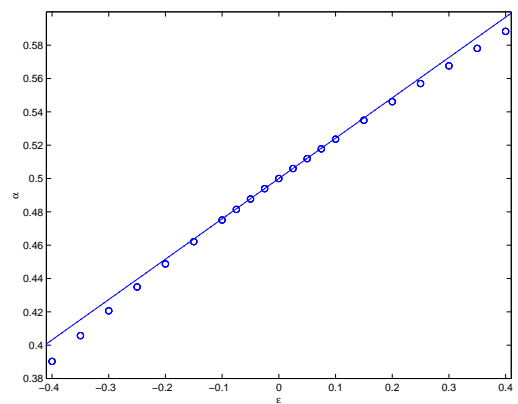

Figure 6: Relation between $\alpha$ and $\epsilon$ for Barenblatt's equation. The continuous curve is the first-order approximation for $\alpha(\epsilon)$. The circle points are the results of our simulations.

compact support) initial conditions. Figure 4 shows the convergence of the prefactors $A_{n}$ to the theoretical values $A$ (dotted lines). The latter depend on the mass of the initial condition. 
We also verified that the profile function is equal to the Gaussian distribution, in agreement with the analytical results. Finally, in Figure 5 we plot the relative difference between successive profiles, $\left\|f_{n}-f_{n-1}\right\| /\left\|f_{n}\right\|$, as a function of the number $n$ of RG iterations. The convergence of this difference to zero can be used as a diagnosis of the self-similar behavior of the long-time asymptotics; it can also be used to check the convergence of the procedure and as a practical stopping criterion for the number of $\mathrm{RG}$ iterations.

The remaining figures, Figures [7-10, illustrate various aspects of the asymptotics of the solutions to the IVP (2.1) and corroborate our Conjecture 2.1. In all simulations performed, $\alpha_{n}$ was observed to converge quickly to $\alpha=\frac{1}{2}$, as the number of RG iterations increases. In order to verify the universality of this fact, we varied: initial conditions (Figure [7); nonlinear perturbations (Figure 8); values of $\mu$ and periodic functions (Figure 9). Figure 10 shows the convergence of the prefactors $A_{n}$ and their nonuniversality. We emphasize that in all simulations, the convergence of the relative difference $\left\|f_{n}-f_{n-1}\right\| /\left\|f_{n}\right\|$ (in both $L^{1}$ and $L^{\infty}$ norms) to zero was observed, as the number of RG iterations increased, thus providing strong evidence of the self-similar nature of the asymptotics.

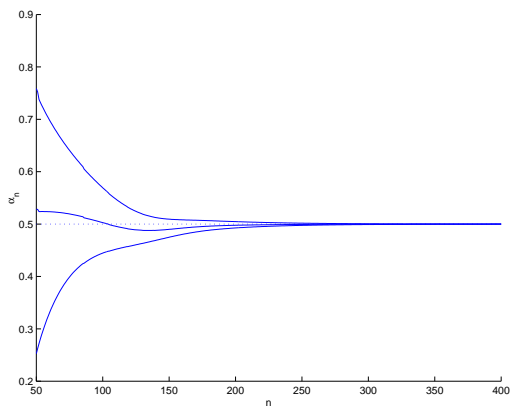

Figure 7: Plot of $\alpha_{n} \times n$, for simulations 1,2 and 4, verifying that $\alpha$ is universal with respect to the initial condition.

Finally, the effect of the term $\mu g$ on the diffusion coefficient is illustrated in Figures 11 and 12. These figures display the points $\left(-\log \phi_{*},-\log \phi\right)$, where $\phi$ denotes the profile function $f_{n}$ computed after the last $\mathrm{RG}$ iteration and $\phi_{*}(x)=\exp \left(-x^{2} / 4\right)$. It is clear that for each simulation these points lie on a straight line. This implies that the computed profiles $\phi$ are the "renormalized Gaussians" $\phi_{\sigma}(x)=\exp \left(-x^{2} / 4 \sigma\right)$, with $\sigma$ being given by the slope of the corresponding straight line. The theoretical values for $\sigma$ are also plotted (stars) in these figures and they are obtained from the explicit computation of the harmonic mean of $1+\mu g$. 


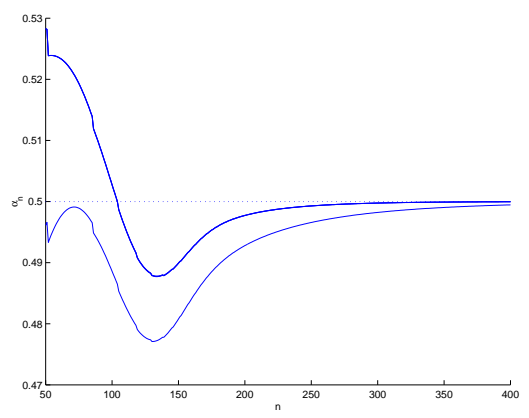

Figure 8: Plot of $\alpha_{n} \times n$, for simulations 1, 5, 6, 14 and 15, verifying that $\alpha$ is universal with respect to the addition of nonlinear terms.

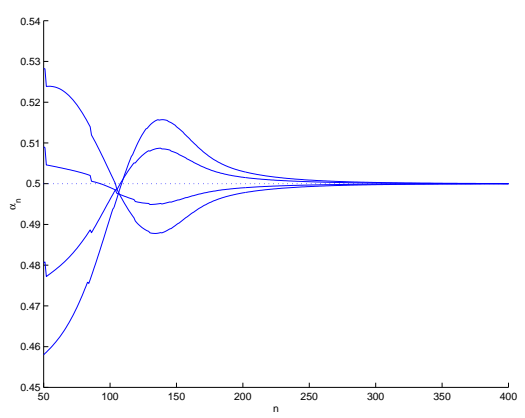

Figure 9: Plot of $\alpha_{n} \times n$, for simulations 1, 3, 7 and 9, verifying that $\alpha$ is universal with respect to the periodic perturbation $g(\cdot)$ and the parameter $\mu$.

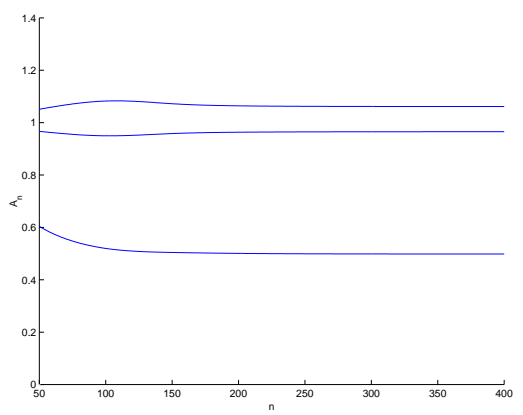

Figure 10: Plot of $A_{n} \times n$, for simulations 1, 2 and 3, showing that $A_{n}$ does converge as $n \rightarrow \infty$. 


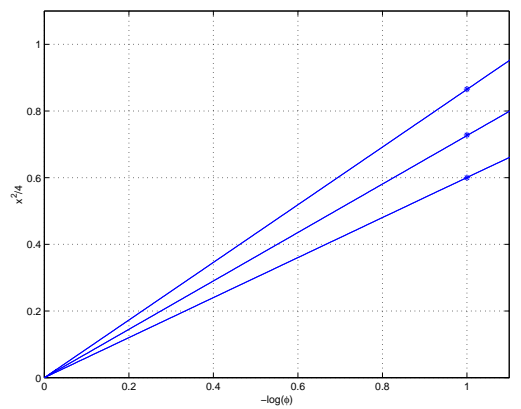

Figure 11: Plot of $-\log \left(\phi_{*}\right) \times-\log (\phi)$, for simulations 8, 10 and 15. The stars plotted are the theoretically computed values for $\sigma$.

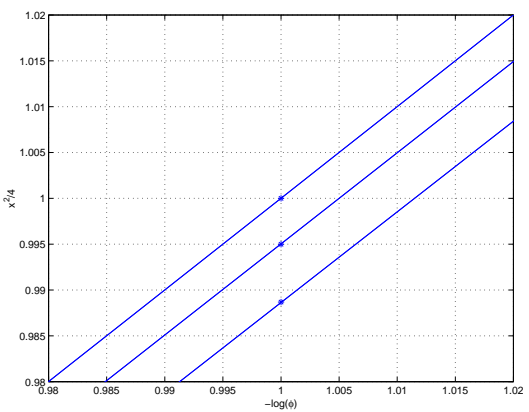

Figure 12: Plot of $-\log \left(\phi_{*}\right) \times-\log (\phi)$, for simulations 1, 3 and 11. The stars are the explicitly calculated $\sigma$.

\section{Concluding Remarks}

From the numerical simulations we have a solid basis that indicates the correctness of our Conjecture 2.1. Furthermore, using the numerical algorithm of Section 4, we have some preliminary results for the dynamics for the following equation (which is in divergence form):

$$
u_{t}=\partial_{x}\left([1+\mu g(x)] \partial_{x} u\right)+\lambda F\left(u, u_{x}, u_{x x}\right) \quad x \in \mathbb{R}, t>1 .
$$

Equations (2.1) and (6.1) differ by a multiple of $g^{\prime}(x) u_{x}$. Our results indicate that this term is irrelevant in the RG sense even though the term $u_{x}$ is relevant. When $F=u^{q} u_{x}$ in equation (6.1), our conjecture has been proved [9].

The version of the numerical procedure that we described here is not appropriate for the analysis of the dynamics when marginal perturbations of the type $u u_{x}$ are included. Marginal perturbations have been handled successfully using a modified version of the procedure in [13]. 
To verify the strength and flexibility of the method, we also performed a numerical study of Equation (2.1) with $\lambda F=-u^{a}, 1<a<3$. First suppose $\mu=0$ in Eq. (2.1). In this case, the Laplacian and the nonlinear term $\lambda F$ are equally important asymptotically. The choice $\beta_{n}=\frac{1}{2}$ keeps the Laplacian invariant to scaling. As discussed in Section 4 , we expect that $\alpha_{n}$ will converge to $\alpha=\frac{1}{a-1}$ as $n \rightarrow \infty$, so that the nonlinear term remains in the limiting equation describing the long-time asymptotic behavior. This result is rigorous and it was first proved by Brezis, Peletier and Terman [5] and also verified by Bricmont et al. [6, 7] using the RG approach. When $\mu \neq 0$, we expect that the same scalings will hold. These scalings lead to the conjecture (2.4), which we have verified numerically. In Figure 13 we plot the curve $\alpha=\frac{1}{a-1}$ together with the results of our simulations. The renormalization of the profile function by a factor of $\sqrt{\sigma}$ in the argument has been verified: we have plotted the computed profile function against $x / \sqrt{\sigma}$, where the factor $\sigma$ was computed a priori as the harmonic mean of $1+\mu g(x)$, and the plot coincides with the plot of the computed profile function with $\mu=0$.

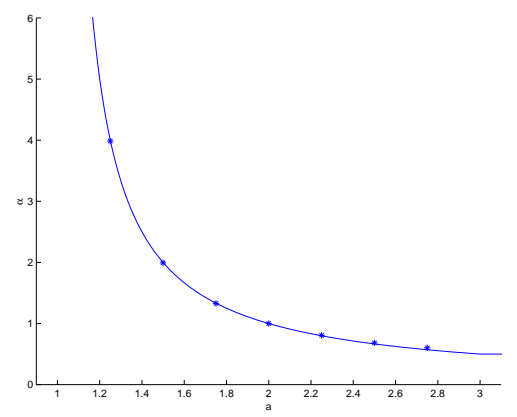

Figure 13: Relation between $\alpha$ and $a$ for $\lambda F(u)=-u^{a}$, with $1<a<3$. The continuous curve is obtained theoretically and the stars are the results of our simulations.

Acknowledgments L.R. and J.M. thank CNPq-Brazil for providing them with scholarships at the undergraduate and graduate levels, respectively. F.F. thanks the Departamento de Matemática - UFMG for their hospitality during his visit in July of 2002.

\section{References}

[1] G. I. Barenblatt, Scaling, self-similarity and intermediate asymptotics, Cambridge University Press, Cambridge, 2 ed., 1996.

[2] A. Bensousan, J. Lions, and G. Papanicolaou, Asymptotic Analysis of Periodic Structure, North Holland, Amsterdam, 1978. 
[3] M. Berger AND R. Kohn, A rescaling algorithm for the numerical calculation of blowing-up solutions, Comm. Pure Appl. Math., 41 (1988), pp. 841-863.

[4] G. A. Braga, F. Furtado, And V. Isaia, Smoothing of barenblatt equation singularity: a numerical and analytical renormalization group study. In preparation.

[5] H. Brezis, L. A. Peletier, And D. Terman, A very singular solution of the heat equation with absorption, Archive Rat. Mech. Anal., 95 (1986), pp. 185-209.

[6] J. Bricmont And A. Kupiainen, Renormalizing partial differential equations, Constructive Physics, 446 (1995), pp. 83-115.

[7] J. Bricmont, A. Kupiainen, And G. Lin, Renormalization group and asymptotics of solutions of nonlinear parabolic equations, Communications in Pure and Applied Mathematics, 47 (1994), pp. 893-922.

[8] L. Chen And N. Goldenfeld, Numerical renormalization group calculations for similarity solutions and travelling waves, Physical Review E, 51 (1995), pp. 55775581.

[9] G. Duro AND E. ZuAZuA, Large time behavior for convection-diffusion equations in rn with periodic coefficients, Journal of Differential Equations, 167 (2000), pp. 275-315.

[10] G. Fibich, W. Ren, And X. P. Wang, Stability of solitary waves for nonlinear schrödinger equations with inhomogeneous nonlinearities, Phys. D, 175 (2003), pp. 96-108.

[11] M. Gell-Mann And F. E. Low, Quantum electrodynamics at small distances, Phys. Rev., 95 (1954), pp. 1300-1312.

[12] N. Goldenfeld, Lectures on Phase Transitions and the Renormalization Group, Addison-Wesley, Reading, 1992.

[13] V. IsAIA, Intermediate Asymtotic Behavior of Nonlinear Parabolic PDEs via a Renormalization Group Approach: a Numerical Study, PhD thesis, Department of Mathematics, University of Wyoming, Laramie, Wyoming, 2002.

[14] S. K. MA, Modern Theory of Critical Phenomena, Benjamin/Cummings Publishing Company, Reading, 1976.

[15] D. W. Mclaughlin, G. C. Papanicolaou, C. Sulem, and P. L. Sulem, Focusing singularity of the cubic schrödinger equation, Phys. Rev. A, 34 (1986), pp. $1200-1210$. 
[16] A. Naddaf And T. Spencer, On homogenization and scaling limit of some gradient perturbations of a massless free field, Comm. Math. Phys., 183 (1997), pp. 55-84.

[17] W. Ren And X. P. WANG, An iterative grid redistribution method for singular problems in multiple dimensions, J. Comp. Phys., 159 (2000), pp. 246-273.

[18] K. Wilson, Renormalization group and critical phenomena $i$, ii, Phys. Rev. B, 4 (1971), pp. 3174-3183, 3184-3205. 